\title{
An Analysis of the Applications of Natural Language Processing in Various Sectors
}

\author{
Priya $\mathrm{B}^{\mathrm{a}, 1}$, Nandhini J.M $\mathrm{M}^{\mathrm{b}}$ and Gnanasekaran $\mathrm{T}^{\mathrm{c}}$ \\ ${ }^{a, 1}$ Associate Professor, Dept of CSE, Sri Sai Ram Engineering College, Chennai, India \\ ${ }^{b}$ Associate Professor, Dept of IT, Sri Sai Ram Institute of Technology, Chennai, India \\ ${ }^{c}$ Professor, R.M.K Engineering College,Chennai, India
}

\begin{abstract}
Natural Language processing (NLP) dealing with Artificial Intelligence concept is a subfield of Computer Science, enabling computers to understand and process human language. Natural Language Processing being a part of artificial intelligence provides understanding of human language by computers for the purpose of extracting information or insights and create meaningful response. It involves creating algorithms that transform text in to words labeling With the emerging advancements in Machine learning and Deep Learning, NLP can contributed a lot towards health sector, education, agriculture and so on. This paper summarizes the various aspects of NLP along with case studies associated with Health Sector for Voice Automated System, prediction of Diabetes Millets, Crop Detection technique in Agriculture Sector.
\end{abstract}

Keywords. Machine Learning, Deep Learnig, Artificial Neural Network

\section{Introduction}

NLP processes human language and produces computer understandable output or human understandable response as part of artificial intelligence application/implementation. NLP refers to a machine's ability to answer in a language that a human can understand. It is possible to build algorithms that convert text into words. The words can be classified based on their meanings. To extract meaning from human languages, most NLP techniques rely on machine learning. Linguistics and Computer Science are the two fields of NLP. Linguistics is the study of language, including its structure, grammar, meaning, and various types of phrases One of the fastest and far reaching emerging technology in Computer Science is the study of linguistics or natural language processing covering overlapping subject areas that include Machine Learning, Deep Learning and Artificial Intelligence.

\footnotetext{
${ }^{1}$ Priya. B,Associate Professor, Dept of CSE, Sri Sai Ram Engineering College, Chennai, India. E-mail:bpriya.cse@sairam.edu.in
} 


\section{Build Data Model}

NLP spans over several levels and frequently these different levels integrate with one another as shown in Figure 1.

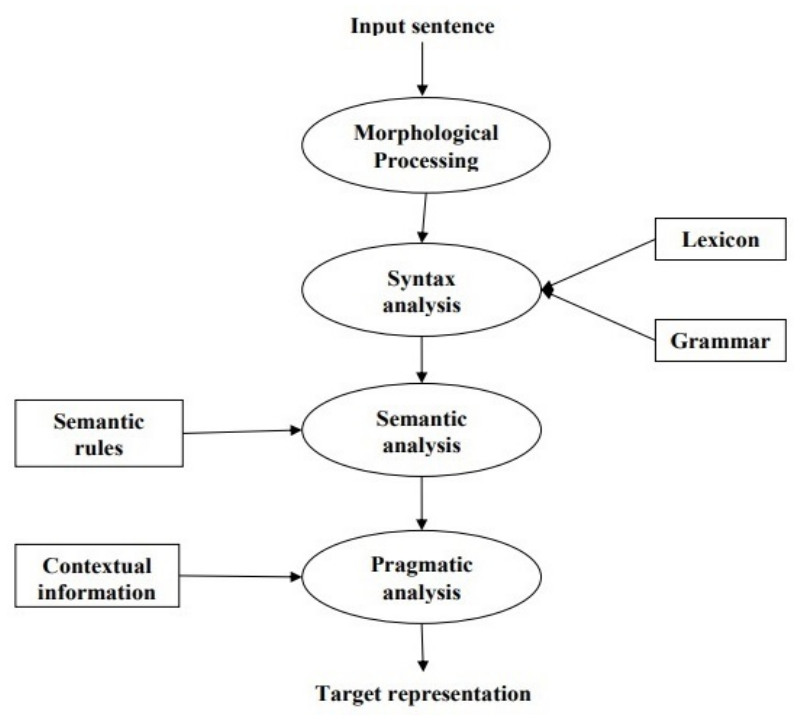

Figure 1. Levels of NLP

The various levels are

1. Morphological level: This level concerns with the study of word structures and formation of words, concentrating on the analysis of the individual components of words.

2. Lexical Level: The Lexical deals with the words and their lexical meaning. The processing at this level uses individual lexemes, an abstract unit of morphological analysis.

3. Syntactic Level: At the Syntactic level the output of the lexical analysis is used to cluster words into phrase and clause brackets. Phrase extraction is done at this level Semantic Level: This level deals with the real meaning of the sentence by relating its syntactic features and disambiguating words with multiple definitions. It implicates the proper explanation of the meaning of sentences.

4. Pragmatic Level: The pragmatic level of linguistic analysis is concerned with the application of real-world experience and the comprehension of how this affects the communication meaning. A more detailed representation is obtained by analysing the contextual dimension of the documents and queries. 


\section{Challenges in NLP}

In The complication of NLP is due to the essence of human language. The rules used for information communication using natural languages are not so easy for the computers as it is not easily understandable by it. Some rules can be high-leveled and abstract and some rules can be low-leveled. In wider sense, to understand the words of human language and its concept becomes essential to deliver the intended message. Natural language processing is difficult for computers to implement due to its complexity and imprecise features, although humans can easily master it.

\section{Machine Learning and NLP}

Machine Learning algorithms and Artificial Intelligence are used in Machine learning for NLP and text analytics to identify and understand the meaning of text documents. Machine learning and Artificial Intelligence has a major place in natural language processing and text analytics by improving, accelerating and automating the underlying text analytics functions as depicted in Figure 2.

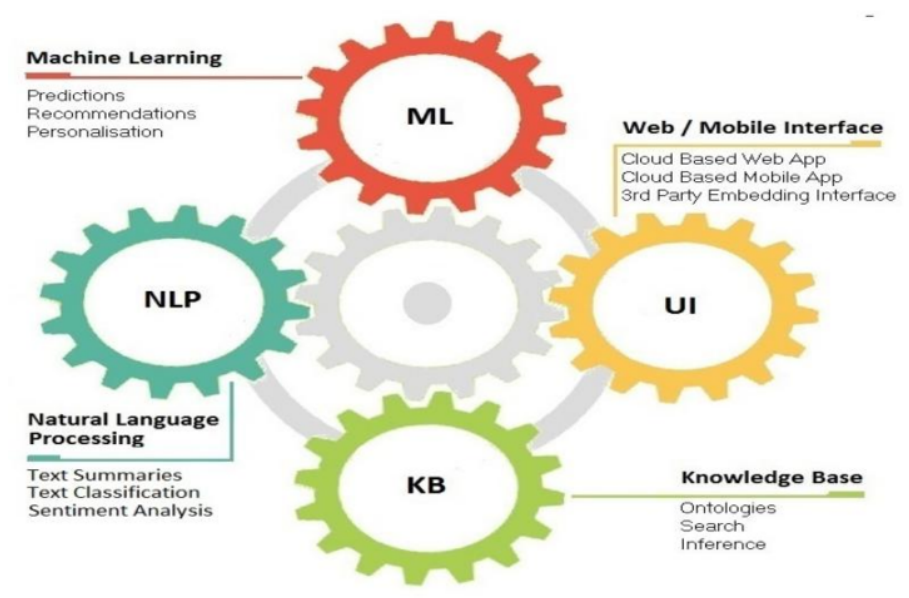

Figure 2: Machine Learning and NLP

The features of NLP can change the unstructured data into useable data. A machine learning model learns and updates from its training data. Machine learning for NLP and text analytics comprises a set of statistical techniques such as entities, sentiments and speech identification. ML and NLP have some overlay, as Machine Learning is often used for NLP tasks.

\section{Deep Learning and NLP}

Deep learning for NLP is a branch of artificial intelligence that aids the computer in manipulating, and interpreting human language. Development of Computational algorithms in NLP is used for analysing and representing human languages using 
machine learning and algorithmic approaches. Neural networks are the basis of Deep learning. Deep learning algorithms helps to solve problems in Natural Language Processing (NLP) that machine learning algorithms couldn't solve. The following are some of the most popular NPL applications that are made easier to solve with deep learning are

1. Text Classification and Categorization: In current scenario, text classification becomes important for many applications like web searching, email spam filtering, language identification, etc.

2. Named Entity Recognition (NER) : Named Entity Recognition (NER) is the first step for information extraction and entity classification.

3. Question Answering: The major applications of Natural Language Processing research is Question Answering. This application still remains as a challenge especially for search engines. NLP is used to create an interactive interface between human and computer. This is one of the most advanced features of deep learning-based NLP, in which people use a computer to find the answer to a specific question from a given text as input. This application would also improve website automated chat

\section{Applications of NLP}

Natural Language Processing is used in many fields. Many complex innovative applications using cutting edge technology falls under this category. The various sectors in which NLP is applied are given in Figure 3.

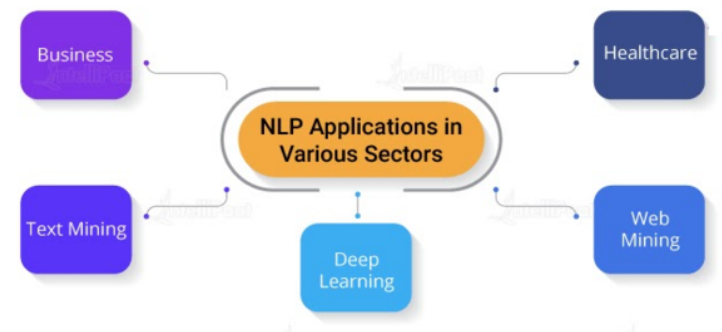

Figure 3: Application Sectors of NLP

Some of the popularly used NLP applications are:

- Machine Translation: The amount of knowledge available on the internet is growing at an exponential pace. NLP plays a significant role in meeting these requirements. By translating technical manuals, support documents, or catalogues at a significant expense, machine translation helps us resolve language barriers that are often encountered. The difficulty with machine translation technology isn't in interpreting sentences, but in comprehending them.

- Automatic summarization: Information overload is the real challenge, when a user has to access relevant information from a large knowledge base. Automatic summarization can be used to summarise the meaning of documents and records, as well as to comprehend the emotional meanings contained within the data. It's typically used to provide a high-level summary of a news storey or a series of blog posts while avoiding rhyming. 
- Sentiment Analysis: NLP plays a vital role in monitoring social media. It's commonly used in sentiment analysis for a variety of purposes. The aim of sentiment analysis is to identify sentiments through several posts, or even within the same post, where emotion isn't always expressed directly. Many businesses use sentiment analysis to identify consumer views and sentiment online in order to help them develop better products.

- Health Care: Natural language processing helps to improve the completeness and accuracy of Electronic Health Records by transforming free text into standardized data. They examine patients' data to assess the implications of phenotyping, which is beneficial to doctors. Algorithms focused on natural language processing (NLP) identify possible errors in healthcare delivery. The NLP predictive analysis aids in the identification of high-risk patients, thus improving diagnostic procedures.

- Text Mining: The process of extracting high-quality information from text is known as Text Mining. It is also termed as text data mining. Text recognition, customer care, personalized bots, and sentiment analysis are examples of NLP applications in text mining.

- Education: NLP will parse and summarize arguments. It also assist authors in improving their writing and encouraging them to rewrite essays. Feedback to to learners about the organization can be obtained from NLP. NLP solutions can be combined to create automated writing assessment (AWE) systems that can provide low-level feedback (Example: vocabulary tips) or higher-level feedback (Example, grammar corrections) (e.g., advice about the cohesion of discourse).

- Agriculture: Agriculture is highly important to global economy. With the continued growth of the human population, demand on the agricultural system is increasingly growing. Agri-technology and precision farming, collectively known as digital agriculture, have emerged as new scientific fields that employ data-intensive approaches to boost agricultural productivity while reducing environmental effects. Data obtained from a number of sensors in modern agricultural operations allows for a deeper understanding of the operating environment (an interaction of dynamic crop, soil, and weather conditions) as well as the process itself (machinery data), resulting in more precise and faster decision making.

\section{References}

[1] Sandeep Nigam; Ajit Kumar Das; Rakesh Chandra ,Machine Learning Based Approach To Sentiment Analysis, IEEE Xplore 2019.

[2] Tom Young Devamanyu Hazarika; Soujanya Poria; Erik Cambria, Recent Trends in Deep Learning Based Natural Language Processing, IEEE Computational Intelligence Magazine Volume: 13, Issue: 3, Aug. 2018

[3] M. Chandhana Surabhi, Natural language processing future, IEEE Xplore, 2013

[4] Friedman C, Rindflesch TC, Corn M, Natural language processing: State of the art and prospects for significant progress, Journal of Biomedical Informatics, 25 Jun 2013, 46(5):765-773.

[5] Krishna Prakash Kalyanathaya, D. Akila and P. Rajesh, Advances in Natural Language Processing - A Survey of Current Research Trends, Development Tools and Industry Applications, International Journal of Recent Technology and Engineering (IJRTE) ISSN: 2277- 3878, Volume-7, Issue-5C, February 2019

[6] Denis, Design and Construction of a NLP Based Knowledge Extraction Methodology in the Medical Domain Applied to Clinical Information, Healthcare Informatics Research 2018;24(4): 376-380

[7] Matthew N. O. Sadiku, Yu Zhou, and Sarhan M. Musa, Natural Language Processing, International Journal of Advances in Scientific Research and Engineering (IJASRE), Volume 4, Issue 5, May - 2018 